\title{
A comparative study on the time to achieve negative nucleic acid testing and hospital stays between Danoprevir and Lopinavir/Ritonavir in the treatment of patients with COVID- 19
}

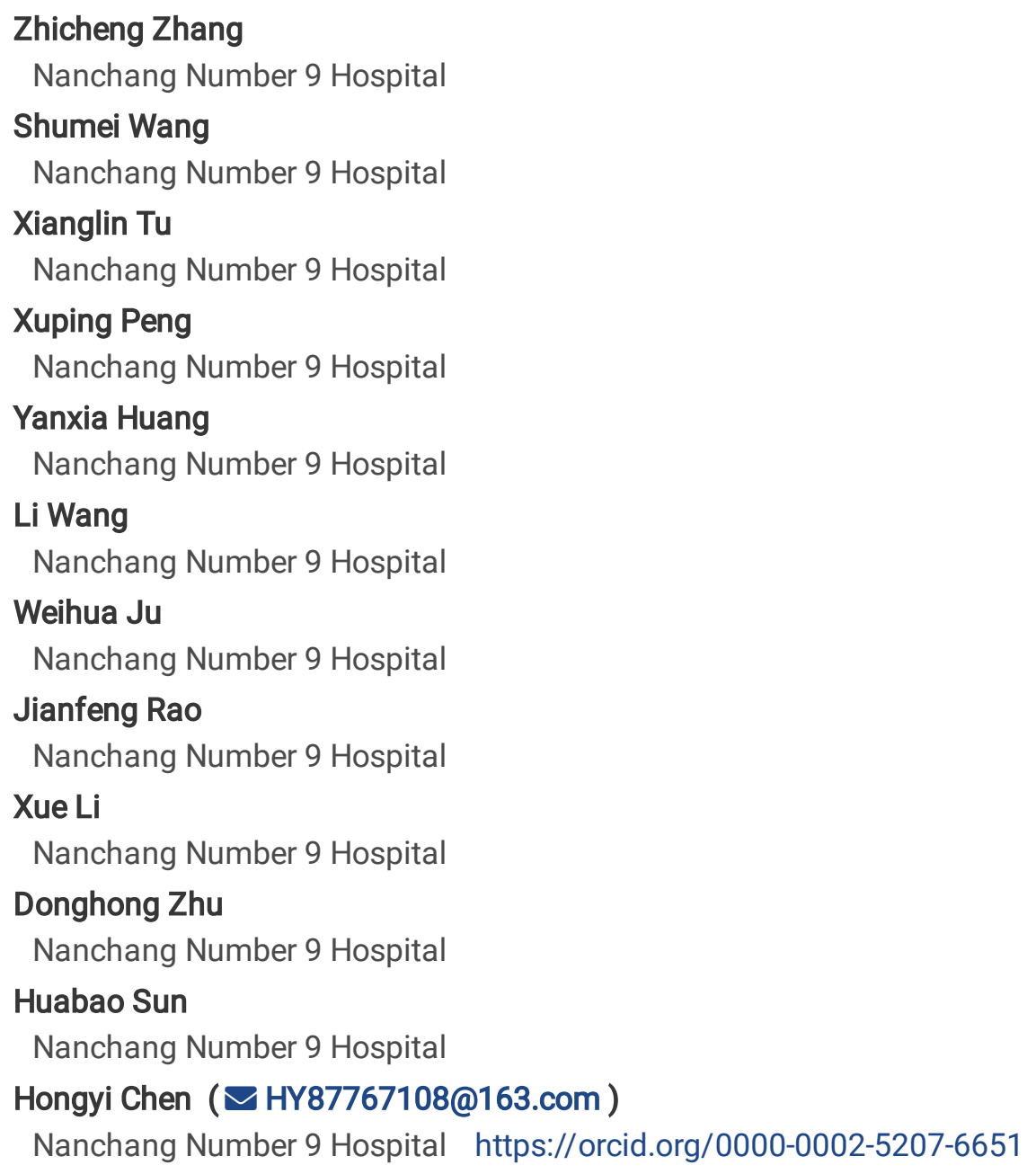

Research article

Keywords: COVID-19, Danoprevir, Lopinavir/Ritonavir, time to achieve negative nucleic acid testing

Posted Date: May 29th, 2020

DOI: https://doi.org/10.21203/rs.3.rs-28376/v1

License: @ (i) This work is licensed under a Creative Commons Attribution 4.0 International License. Read Full License 


\section{Abstract}

\section{Background}

In late December 2019, the coronavirus disease 2019 (COVID-19) first outbroke in Wuhan city, China, and has now become a global pandemic. However, there is no specific antiviral treatment for COVID-19.

Methods

This study was approved by the Ethics Committee of the Ninth Hospital of Nanchang. This study enrolled 33 COVID-19 patients in the nineth hospital of Nanchang from January 27th to February 24th, 2020. Clinical indexes of patients upon admission/discharge were examined. Patients were divided into two groups according to different treatment plans (Danoprevir; Lopinavir/Ritonavir). The days to achieve negative nucleic acid testing and the days of hospital stays were counted and statistically analyzed.

Results

COVID-19 patients treated with Danoprevir or Lopinavir/Ritonavir were all improved and discharged. Indexes like blood routine, inflammation and immune-related indexes were significantly recovered after treatment. Additionally, under the circumstance that there was no significant difference in patients' general information between the two groups, we found that the mean time to achieve negative nucleic acid testing and hospital stays of patients treated with Danoprevir both were significantly shorter than those of patients with Lopinavir/Ritonavir.

\section{Conclusion}

Collectively, applying Danoprevir is a good treatment plan for COVID-19 patients.

Highlight

1. The average time to achieve negative nucleic acid testing of COVID-19 patients treated with Danoprevir was obviously shorter than that of patients treated with Lopinavir/Ritonavir;

2. The average time of hospital stays of COVID-19 patients treated with Danoprevir was significantly shorter than that of patients treated with Lopinavir/Ritonavir.

\section{Background}

Coronavirus disease 2019 (COVID-19) is caused by a novel severe acute respiratory syndrome coronavirus (SARS-CoV-2), a causative agent of a potentially fatal disease, and it has become a great global public health concern ${ }^{1}$. Form late 2019 to early 2020 , the novel coronavirus SARS-CoV-2 suddenly broke out among ordinary people in Wuhan city, China, and rapidly spread in a short period of time. According to the data released by the World Health Organization (WHO), more than 2,540,000 cases were reported worldwide by April 23, 2020, among which 175,000 patients died. The massive outbreak of COVID-19 has caused numerous casualties and huge economic loss. Therefore, it is vital to make effective treatment plans as soon as possible and speed up the coordination of prevention and treatment, so as to protect people's health and reduce economic loss.

The symptoms of COVID-19 infections occur after an average incubation period of approximately 5.2 days ${ }^{2}$. The most common clinical symptoms include fever, dry cough, myalgia, fatigue, dyspnea, and diarrhea ${ }^{3}$, which are generally similar to the symptoms caused by $\beta$-coronavirus ${ }^{4}$. Studies have indicated that clinical indexes such as patients' blood indexes are important indicators essential for the diagnosis and treatment of COVID-19. In patients with COVID-19, there is an increase in white blood cell (WBC) count and plasma proinflammatory cytokine level. For example, a prior study showed that the C- 
reactive protein (CRP) of a COVID-19 patient was $16.16 \mathrm{mg} / \mathrm{L}$, which was above the normal range (0-10 mg/L), with a high erythrocyte sedimentation rate (ESR) and D-dimer level ${ }^{5}$. Besides, studies found that the lymphocyte count and its percentage were significantly decreased in severe COVID-19 patients relative to those in mild patients ${ }^{4,6}$. Moreover, the levels of cytokines and chemokines in the blood of COVID-19 patients were significantly elevated, while some severe COVID-19 patients showed high levels of pro-inflammatory cytokines, such as IL2, IL7, IL10, GCSF, IP10, MCP1, MIP1a and TNFa ${ }^{4}$. Collectively, screening out the indicators with significant differences is of great reference value for the diagnosis and treatment of COVID-19 patients.

At present, there have been no specific antiviral drugs or vaccines available for treatment of patients with COVID-19. Therefore, it is desirable to develop appropriate and effective therapeutic drugs. A study has revealed that broad-spectrum antiviral drugs and HIV protease inhibitors could attenuate the viral infection ${ }^{7}$. Antiviral therapies have been applied in patients currently. For example, patients in a study were given $75 \mathrm{mg}$ oseltamivir, $500 \mathrm{mg}$ lopinavir, $500 \mathrm{mg}$ ritonavir and $0.25 \mathrm{~g}$ ganciclovir twice a day for 3-14 days ${ }^{8}$. The in vitro experiments done by Wang $\mathrm{M}$ et al. found that Remdesivir and Chloroquine play inhibitory roles in the growth of SARS-CoV- ${ }^{9}$, and preliminary clinical treatment in COVID-19 patients showed that Chloroquine was an effective drug in improving clinical efficacy and potentiating virus clearance ${ }^{10}$. Danoprevir is a potent hepatitis $C$ virus $(\mathrm{HCV})$ protease inhibitor (NS3/4A, IC50 $=0.29 \mathrm{nM})$, and was approved and marketed in China in 2018 for the treatment of patients with chronic hepatitis C. The latest researches suggested that patients $(n=11)$ who underwent 4-12 days treatment of Danoprevir and Ritonavir were all discharged ${ }^{11}$. In addition, studies indicated that Lopinavir/Ritonavir (LPV/r) manifested favorable effect in fighting against COVID-19 virus and relieving clinical symptoms $^{12}$, and combined treatment of traditional Chinese medicine and western medicine could protect COVID-19 patients from severe kidney damage ${ }^{13}$. However, the efficacy of Danoprevir and LPV/r in the treatment of COVID-19 have not been compared.

In this study, on the basis of the treatment plan and the recovery of 33 COVID-19 patients in the nineth hospital of Nanchang, we analyzed the clinical conditions of patients treated with Danoprevir and LPV/r and made a comparative study concerning the time to achieve negative nucleic acid testing (NAT) and hospital stays between the two groups, so as to determine the efficacy of the two therapeutic drugs.

\section{Methods}

\subsection{Research Object}

A total of 33 COVID-19 patients in the nineth hospital of Nanchang from January 27 to February 24, 2020 were involved in this study, including 11 males and 22 females aged between 18-71. Nucleic acid samples were collected from the respiratory tract of patients and were confirmed to be positive by quantitative PCR. The information was detailed in Table 2 .

\subsection{Clinical classification and treatment regimen}

The severity of disease was distinguished according to the clinical features of COVID-19 patients: 1. mild type: the clinical symptoms are mild, and no signs of pneumonia are found on imaging; 2. moderate type: having fever and respiratory symptoms, and signs of pneumonia can be observed on imaging; 3 . severe type: adults who meet any of the following criteria: respiratory rate $\geq 30$ times /min, oxygen saturation in resting state $\leq 93 \%$; arterial partial pressure of oxygen (PaO2) /concentration of oxygen (FiO2) $\leq 300 \mathrm{mmHg}$; pulmonary imaging shows significant progression of lesion $>50 \%$ within $24-$ 48 hours.

All patients were classified into mild, moderate and severe types, and then grouped into Danoprevir group and LPV/r group according to the therapeutic regimen based on the actual situation of patients.

\subsection{Evaluation criteria}


(1) Blood routine index: WBC (4-10 $\left.\times 10^{9} / \mathrm{L}\right)$; lymphocyte count $\left(0.8-4.0 \times 10^{9} / \mathrm{L}\right)$; eosinophilic granulocyte count (EOC, $0.05-$ $0.5 \times 10^{9} / \mathrm{L}$ ); ferritin (male: $16-220 \mathrm{ng} / \mathrm{mL}$, female: $\left.10-124 \mathrm{ng} / \mathrm{mL}\right)$; D-dimer $(0-0.6 \mathrm{mg} / \mathrm{L})$.

(2) Inflammation-related indexes: CRP (<10 mg/L); ESR $(0-15 \mathrm{~mm} / \mathrm{h})$.

(3) Immune-related indexes: immunoglobulin G (IgG, 7-16 g/L); interleukin-4 (IL-4, 0-2.8 pg/mL); interleukin-6 (IL-6, 0$5.3 \mathrm{pg} / \mathrm{mL}$ ); interleukin-10 (IL-10, 0-4.91 pg/mL); CD8+\% (15-44); CD4+\% (27-51); CD16+\%CD56+\% (7-40).

(4) Negative NAT detected by PCR.

(5) The hospital days of patients.

\subsection{Statistical analysis}

All data were processed by Graphpad Prism 6.0 software (Graphpad Prism, San Diego, CA, USA). Enumeration data of patients in the two groups were analyzed by Fisher exact test, while measurement data were presented as mean \pm standard deviation and were analyzed by $t$ test. $P<0.05$ was considered statistically significant.

\section{Results}

\subsection{Comparison of clinical indexes upon admission and discharge}

In this study, we enrolled 33 COVID-19 cases in the nineth hospital of Nanchang, and nucleic acid samples were collected from the respiratory tract of patients and confirmed to be positive. We statistically analyzed the blood routine and immunerelated indexes of the patients on admission/discharge for the purpose of direct comparison of therapeutic outcomes. As shown in Table 1, the abnormal proportions (the percentage of patients with abnormal clinical indexes in total number of patients) of clinical indexes were evidently lower in patients on discharge than those in patients on admission. Besides, several indexes (WBC count, lymphocyte count, CRP, IL-6, IL-10, and CD4+\%) of patients on admission and discharge showed a significant difference $(P<0.05)$. Blood routine and some immune indexes are commonly used for disease diagnosis, as they can effectively determine the patients' physical conditions. Based on the clinical data, it turned out that most indexes of patients recovered to normal after treatment, suggesting that our treatment had favorable outcomes. Collectively, most blood routine and immune-related indexes recovered to normal in patients who could be discharged. 
Table 1

Comparison of clinical indexes upon admission and discharge

\begin{tabular}{|c|c|c|c|c|c|c|c|c|c|}
\hline \multirow[t]{2}{*}{ Indexes } & \multicolumn{4}{|c|}{ Admission } & \multicolumn{4}{|c|}{ Discharge } & \multirow[t]{2}{*}{$P$ value } \\
\hline & Case & Normal & Abnormal & $\begin{array}{l}\text { Abnormal } \\
\text { proportion }\end{array}$ & Case & Normal & Abnormal & $\begin{array}{l}\text { Abnormal } \\
\text { proportion }\end{array}$ & \\
\hline WBC count & 33 & 20 & 13 & 0.3939 & 32 & 28 & 4 & 0.1250 & $0.0227 *$ \\
\hline $\begin{array}{l}\text { Lymphocyte } \\
\text { count }\end{array}$ & 33 & 19 & 14 & 0.4242 & 32 & 28 & 4 & 0.1250 & $0.0116^{*}$ \\
\hline ESO count & 33 & 8 & 25 & 0.7576 & 33 & 14 & 19 & 0.5758 & 0.1912 \\
\hline CRP & 26 & 9 & 17 & 0.6538 & 16 & 14 & 2 & 0.1250 & $0.0012^{*}$ \\
\hline Ferritin & 20 & 13 & 7 & 0.3500 & 13 & 13 & 0 & 0.0000 & / \\
\hline ESR & 23 & 2 & 21 & 0.9130 & 10 & 4 & 6 & 0.6000 & 0.0534 \\
\hline $\operatorname{lgG}$ & 22 & 13 & 9 & 0.4091 & 14 & 7 & 7 & 0.5000 & 0.7343 \\
\hline D-dimer & 27 & 10 & 17 & 0.6296 & 19 & 8 & 11 & 0.5789 & 0.7670 \\
\hline IL-4 & 31 & 0 & 31 & 1.0000 & 18 & 5 & 13 & 0.7222 & / \\
\hline IL-6 & 31 & 4 & 27 & 0.8710 & 18 & 9 & 9 & 0.5000 & $0.0074 *$ \\
\hline IL-10 & 31 & 3 & 28 & 0.9032 & 18 & 7 & 11 & 0.6111 & $0.0255^{\star}$ \\
\hline CD8+\% & 20 & 18 & 2 & 0.1000 & 16 & 15 & 1 & 0.0625 & 1.0000 \\
\hline CD4+\% & 22 & 6 & 16 & 0.7273 & 16 & 13 & 3 & 0.1875 & $0.0025^{\star}$ \\
\hline CD16+\%CD56+\% & 17 & 11 & 6 & 0.3529 & 14 & 10 & 4 & 0.2857 & 1.0000 \\
\hline \multicolumn{10}{|c|}{ 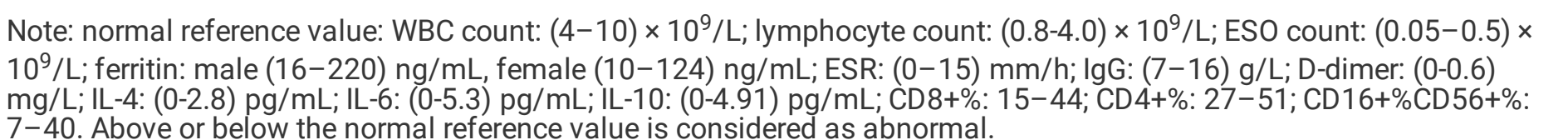 } \\
\hline
\end{tabular}

\subsection{Comparison of general information between the two groups}

Five cases out of 33 COVID-19 patients were given Danoprevir, and 28 cases out of 33 COVID-19 patients were given LPV/r. Our study included demographic information, clinical features on admission, underlying medical disease and clinical classification on admission (Table 2). Most COVID-19 patients showed some clinical features upon admission, among which fever (58\%) and cough (61\%) were the most common symptoms. Symptoms like chest distress, dyspnoea and fatigue mainly appeared in patients over 40 years. On the basis of clinical diagnosis, patients were divided into mild type, moderate type and severe type. The moderate cases were the main subject of our study. In order to compare the efficacy of Danoprevir and LPV/r in treating COVID-19, we performed statistical analysis on the general information of the patients in the two groups. In terms of significance, there was no significant difference $(P>0.05)$ in patients' general information between the two groups, which is conducive to the subsequent assessment of the efficacy of two drugs. 
Table 2

General information of patients in the two groups

\begin{tabular}{|c|c|c|c|c|}
\hline & & Danoprevir & $\mathrm{LPV} / \mathrm{r}$ & $P$ value \\
\hline Cases & & 5 & 28 & \\
\hline \multicolumn{5}{|c|}{ Demographic information } \\
\hline Age (median) & & 44 & 43 & 0.7620 \\
\hline \multirow[t]{2}{*}{ Gender (\%) } & Male & $2(40.0)$ & $9(32.1)$ & 1.0000 \\
\hline & Female & $3(60.0)$ & $19(67.9)$ & \\
\hline \multicolumn{5}{|l|}{ Clinical features } \\
\hline \multirow[t]{2}{*}{ Asymptomatic (\%) } & Yes & $3(60.0)$ & $10(35.7)$ & 0.3600 \\
\hline & No & $2(40.0)$ & $18(64.3)$ & \\
\hline \multirow[t]{2}{*}{ Fever $(\%)$} & Yes & $1(20.0)$ & $13(46.4)$ & 0.3662 \\
\hline & No & $4(80.0)$ & $15(53.6)$ & \\
\hline \multirow[t]{2}{*}{ Cough (\%) } & Yes & $1(20.0)$ & $12(42.9)$ & 0.6253 \\
\hline & No & $4(80.0)$ & $16(57.1)$ & \\
\hline \multirow[t]{2}{*}{ Chest distress (\%) } & Yes & $0(0.0)$ & $5(17.9)$ & / \\
\hline & No & $5(100.0)$ & $23(82.1)$ & \\
\hline \multirow[t]{2}{*}{ Dyspnoea (\%) } & Yes & $0(0.0)$ & $1(3.6)$ & / \\
\hline & No & $5(100.0)$ & $27(96.4)$ & \\
\hline \multirow[t]{2}{*}{ Fatigue (\%) } & Yes & $0(0.0)$ & $1(3.6)$ & / \\
\hline & No & $5(100.0)$ & $27(96.4)$ & \\
\hline \multicolumn{5}{|l|}{ Underlying disease } \\
\hline \multirow[t]{2}{*}{ Diabetes (\%) } & Yes & $0(0.0)$ & $4(14.3)$ & / \\
\hline & No & $5(100.0)$ & $24(85.7)$ & \\
\hline \multirow[t]{2}{*}{ Hypertension (\%) } & Yes & $1(20.0)$ & $3(10.7)$ & 0.4996 \\
\hline & No & $4(80.0)$ & $25(89.3)$ & \\
\hline \multirow[t]{2}{*}{ Hepatitis B (\%) } & Yes & $0(0.0)$ & $5(17.9)$ & / \\
\hline & No & $5(100.0)$ & $23(82.1)$ & \\
\hline \multicolumn{5}{|c|}{ Clinical classification on admission } \\
\hline \multirow[t]{3}{*}{ severity (\%) } & Mild & $1(20.0)$ & $9(32.1)$ & / \\
\hline & Moderate & $4(80.0)$ & $18(64.3)$ & \\
\hline & Severe & $0(0.0)$ & $1(3.6)$ & \\
\hline
\end{tabular}
stays between the two groups 
Finally, we analyzed the time to achieve negative NAT and hospital stays of patients in the two groups. All patients in the two groups were monitored until they were discharged. The time for two consecutive negative NAT of throat swab and hospital stays of COVID-19 patients were analyzed and compared. As illustrated in Table 3, it was found that in the Danoprevir group, the first patient got negative NAT on the fourth day, and all patients had a negative result within 13 days, with median time of 7 days and average time of 8 days. While in the LPV/r group, the first patient got a negative NAT result on the fifth day, and all patients had a negative result within 21 days, with the median time of 12 days and average time of 12.5 days. The results suggested that there was no marked difference $(P>0.05)$ in the median time to achieve negative NAT between the two groups, while the average time showed a significant difference $(P<0.05)$. After being treated with two different drugs, the percentage of patients with a positive NAT was significantly decreased $(P<0.05)$ in the Danoprevir group relative to that in the LPV/r group (Fig. 1A). Similarly, the hospital stays of patients in the Danoprevir group ranged from 7-21 days, with the median time of 9 days and the average of 11.4 days. While in LPV/r group, the hospital stays ranged from 8-23 days, with the median and average time of 17 days and 16.7 days, respectively. There was no marked difference $(P>0.05)$ in the median hospital stays of patients in two groups, while the average time showed a significant difference $(P<0.05)$. Moreover, the discharge rate of patients in the Danoprevir group was higher than that in the LPV/r group as shown in Fig. 1B $(P<0.05)$. Taken together, our data demonstrated that the efficacy of Danoprevir was better than that of LPV/r in the treatment of COVID-19.

Table 3

Comparison of time to achieve negative NAT and hospital stays of COVID-19 patients in the two groups

\begin{tabular}{|c|c|c|c|}
\hline & Danoprevir & $\mathrm{LPV} / \mathrm{r}$ & $P$ value \\
\hline Cases & 5 & 28 & \\
\hline \multicolumn{4}{|c|}{ Negative NAT (days) } \\
\hline Median & 7.0 & 12.0 & 0.7903 \\
\hline Average & 8.0 & 12.5 & $0.0388 *$ \\
\hline \multicolumn{4}{|c|}{ Hospital stays (days) } \\
\hline Median & 9.0 & 17.0 & 0.6802 \\
\hline Average & 11.4 & 16.7 & $0.0324^{*}$ \\
\hline
\end{tabular}

\section{Discussion}

Coronaviruses are enveloped signal-stranded RNA viruses that are widely distributed in humans and animals throughout the world ${ }^{4}$. Coronaviruses are one of the major pathogens targeting the human respiratory system. In the past two decades, there were two events similar to COVID-19 pandemic caused by cross-infection between animals with $\beta$-coronavirus and humans. The first instance is SARS emerging in 2002-2003 which caused by a new $\beta$-coronavirus (SARS-CoV) originated from bats. SARS-CoV was transmitted to human with palm civet cats as intermediate hosts in Guangdong province, resulting in a total of 8,422 infectious in Chinese mainland and Hongkong and 916 deaths with a mortality of $11 \%{ }^{14}$. Another one is Middle East Respiratory Syndrome (MERS) in 2012 caused by MERS-CoV, which is also derived from bats. It emerged in Saudi Arabia with camels as intermediate hosts and caused 2,494 infectious and 858 deaths with a mortality of 34\% (https://www.who.int/emergencies/mers-cov/en/. Accessed 16 Feb 2020). SARS-CoV and MERS-CoV are both recognized to be major threat to public health. SARS-CoV-2, which is also originated from bats, manifests variable clinical features from asymptomatic status to acute respiratory distress syndrome and multiple organ dysfunction. The epidemiological and clinical features of COVID-19 suggest a higher transmissivity and a lower mortality rate compared to those of SARS 8,15 . Despite that the overall mortality rate of COVID-19 is lower than SARS or MERS, the mortality rate of 
severe cases with COVID-19 is rather worrying ${ }^{16}$. In today's global pandemic, strict prevention and effective treatment of COVID-19 allow of no delay.

Based on the experience summed up in treating SARS and MERS, anti-viral drugs such as Ribavirin, LPV/r, Arbidol and Remdeswir (a broad-spectrum anti-RNA drug developed for Ebola) have been applied in treatment of COVID-19 patients ${ }^{17,18}$. In the present study, 33 cases were given Danoprevir or LPV/r and grouped. The abnormal proportions of clinical indexes of patients upon discharge were significantly decreased compared to those of patients on admission, suggesting an improved condition of patients. These two kinds of treatment plan had a favorable effect on COVID-19 patients.

Danoprevir is an HCV protease inhibitor which can be used in the treatment of chronic hepatitis C. Ritonavir (RTV) is a CYP3A4 inhibitor that increases blood concentration of Danoreivir. In a study on RTV, 11 COVID-19 patients met the following criteria and could be discharged: (1) normal body temperature for at least 3 days; (2) significantly improved respiratory symptoms; (3) lung imaging showed obvious absorption and recovery of acute exudative lesions; (4) two consecutive negative tests of SARS-CoV-2 nucleic acid ${ }^{11}$. However, there have been no comparisons in the therapeutic effect between Danoprevir and other drugs in the clinical treatment of COVID 19. Lopinavir (LPV), a human immunodeficiency virus 1 (HIV-1) protease inhibitor, usually extends its half-life by inhibiting cytochrome P4507 in combination with RTV. Studies have indicated that LPV at a high dose $(9.6 \mathrm{~g} / \mathrm{mL})$ or a low dose $(5.5 \mathrm{~g} / \mathrm{mL})$ can inhibit SARS-CoV, and it also plays an inhibitory role in the replication cycle of MERS-CoV ${ }^{19,20}$. Although most studies focus on the efficacy of LPV, we also need to be aware the adverse reactions like diarrhea, nausea and weakness which are common in patients. Also there was a report revealing that some patients exhibited elevated total bilirubin, triglyceride and liver enzyme levels after the treatment with $\mathrm{LPV}^{21}$.

In order to better compare the efficacy between Danoprevir and LPV/r in clinical practice and to provide a guidance for clinical treatment of COVID-19 and other pneumonia, we analyzed 33 COVID-19 patients and divided them into two groups (Danoprevir group and LPV/r group). Prior to that, we first analyzed the general information of patients in two groups to exclude the interference of age, gender, underlying diseases, clinical symptoms and other factors (Table 2). Our findings illustrated that the time to achieve two consecutive negative NAT and hospital stays of patients in the Danoprevir group were shorter than those of patients in the LPV/r group, especially the average negative result time and hospital stays $(P<0.05)$.

\title{
4 Conclusions
}

In this study, by analyzing the clinical data of 33 cases (10 mild cases, 22 moderate cases and 1 severe case) in two different treatment groups, we found that Danoprevir produced superior efficacy to LPV/r. In conclusion, our study suggests that it is a correct choice to use Danoprevir in the treatment of patients with COVID19.

\section{Abbreviations}

the coronavirus disease 2019 (COVID-19); severe acute respiratory syndrome coronavirus (SARS-CoV-2); the World Health Organization (WHO); white blood cell (WBC); the C-reactive protein (CRP); erythrocyte sedimentation rate( ESR); hepatitis C virus (HCV); Lopinavir/Ritonavir (LPV/r); nucleic acid testing (NAT); arterial partial pressure of oxygen( PaO2); concentration of oxygen (FiO2); eosinophilic granulocyte count (EOC); immunoglobulin G (IgG); interleukin (IL); Middle East Respiratory Syndrome(MERS); Ritonavir (RTV); Lopinavir (LPV); human immunodeficiency virus 1(HIV-1); RNA-dependent RNA polymerase (RDRP)

\section{Declarations}

\section{Ethics approval and consent to participate}

\author{
Page 8/11
}


This study was approved by the Ethics Committee of the Ninth Hospital of Nanchang. Written informed consent was obtained from individual or guardian participants.

\section{Consent for publication}

All athors consent to submit the manuscript for publication.

\section{Availability of Data and Materials}

Data are available from the authors upon reasonable request.

\section{Competing interests}

The authors declare that they have no potential conflicts of interest.

\section{Funding}

This study was supported in part by grants from the Science and Technology Bureau of Nanchang City.

\section{Author's contributions}

All authors contributed to data analysis, drafting and revising the article, gave final approval of the version to be published, and agreed to be accountable for all aspects of the work.

\section{Acknowledgements}

Not applicable.

\section{References}

1. Rothan HA, Byrareddy SN. The epidemiology and pathogenesis of coronavirus disease (COVID-19) outbreak. J Autoimmun. 2020;109:102433. doi:10.1016/j.jaut.2020.102433.

2. Li Q, et al. Early Transmission Dynamics in Wuhan, China, of Novel Coronavirus-Infected Pneumonia. N Engl J Med. 2020;382:1199-207. doi:10.1056/NEJMoa2001316.

3. Wu YC, Chen CS, Chan YJ. Overview of The 2019 Novel Coronavirus (2019-nCoV): The Pathogen of Severe Specific Contagious Pneumonia (SSCP). J Chin Med Assoc. 2020. doi:10.1097/JCMA.0000000000000270.

4. Huang C, et al. Clinical features of patients infected with 2019 novel coronavirus in Wuhan, China. Lancet. 2020;395:497-506. doi:10.1016/S0140-6736(20)30183-5.

5. Lei J, Li J, Li X, Qi X. CT Imaging of the 2019 Novel Coronavirus (2019-nCoV) Pneumonia. Radiology. 2020;295:18. doi:10.1148/radiol.2020200236.

6. Chen X, et al. Differences between COVID-19 and suspected then confirmed SARS-CoV-2-negative pneumonia: a retrospective study from a single center. J Med Virol. 2020. doi:10.1002/jmv.25810.

7. Lu H. Drug treatment options for the 2019-new coronavirus (2019-nCoV). Biosci Trends. 2020;14:69-71. doi:10.5582/bst.2020.01020. 
8. Chen N, et al. Epidemiological and clinical characteristics of 99 cases of 2019 novel coronavirus pneumonia in Wuhan, China: a descriptive study. Lancet. 2020;395:507-13. doi:10.1016/S0140-6736(20)30211-7.

9. Wang $\mathrm{M}$, et al. Remdesivir and chloroquine effectively inhibit the recently emerged novel coronavirus (2019-nCoV) in vitro. Cell Res. 2020;30:269-71. doi:10.1038/s41422-020-0282-0.

10. Gao J, Tian Z, Yang X, Breakthrough. Chloroquine phosphate has shown apparent efficacy in treatment of COVID-19 associated pneumonia in clinical studies. Biosci Trends. 2020;14:72-3. doi:10.5582/bst.2020.01047.

11. Chen H, et al. First Clinical Study Using HCV Protease Inhibitor Danoprevir to Treat Naïve and Experienced COVID-19 Patients. doi:10.1101/2020.03.22.20034041 (2020).

12. Wang Z, Chen X, Lu Y, Chen F, Zhang W. Clinical characteristics and therapeutic procedure for four cases with 2019 novel coronavirus pneumonia receiving combined Chinese and Western medicine treatment. Biosci Trends. 2020;14:648. doi:10.5582/bst.2020.01030.

13. Li L. The novel coronavirus SARS-CoV-2: high pathogenecity and its prevention and therapy. Basic Clinical Medicine 40, doi:10.16352/j.issn.1001-6325.20200420.002 (2020).

14. 10.1046/j.1440-1843.2003.00518.x Chan-Yeung M, Xu RH. SARS: epidemiology. Respirology 8 Suppl, S9-14, doi:10.1046/j.1440-1843.2003.00518.x (2003).

15. Chan JF, et al. A familial cluster of pneumonia associated with the 2019 novel coronavirus indicating person-to-person transmission: a study of a family cluster. Lancet. 2020;395:514-23. doi:10.1016/S0140-6736(20)30154-9.

16. Li T, Lu H, Zhang W. Clinical observation and management of COVID-19 patients. Emerg Microbes Infect. 2020;9:68790. doi:10.1080/22221751.2020.1741327.

17. Singhal TA, Review of. Coronavirus Disease-2019 (COVID-19). Indian J Pediatr. 2020;87:281-6. doi:10.1007/s12098020-03263-6.

18. Holshue ML, et al. First Case of 2019 Novel Coronavirus in the United States. N Engl J Med. 2020;382:929-36. doi:10.1056/NEJMoa2001191.

19. Yao TT, Qian JD, Zhu WY, Wang Y, Wang GQ. A systematic review of lopinavir therapy for SARS coronavirus and MERS coronavirus-A possible reference for coronavirus disease-19 treatment option. J Med Virol. 2020.

doi:10.1002/jmv.25729.

20. de Wilde AH, et al. Screening of an FDA-approved compound library identifies four small-molecule inhibitors of Middle East respiratory syndrome coronavirus replication in cell culture. Antimicrob Agents Chemother. 2014;58:4875-84. doi:10.1128/AAC.03011-14.

21. Kim UJ, Won EJ, Kee SJ, Jung SI, Jang HC. Combination therapy with lopinavir/ritonavir, ribavirin and interferon-alpha for Middle East respiratory syndrome. Antivir Ther. 2016;21:455-9. doi:10.3851/IMP3002.

\section{Figures}



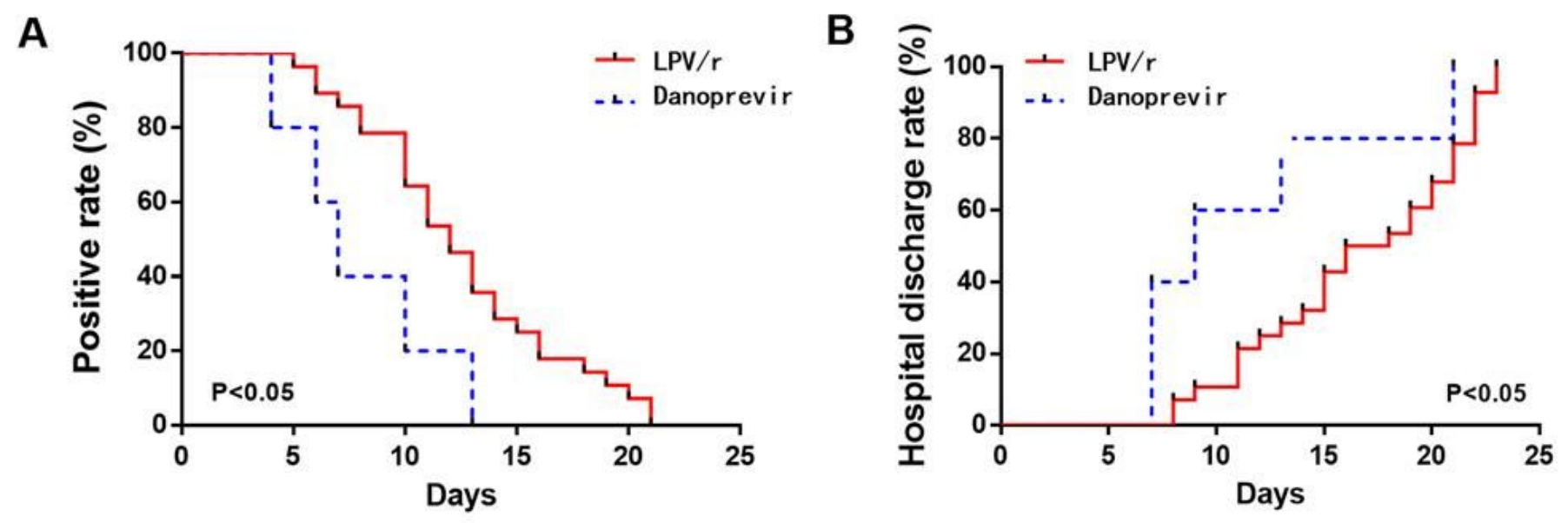

\section{Figure 1}

Comparison of time to achieve negative NAT and hospital stays between the two groups The rate of positive results $(A)$ and the hospital discharge rate (B) of patients in Danoprevir group and LPV/r group were compared; $\mathrm{P}<0.05$ was considered statistically significant. 